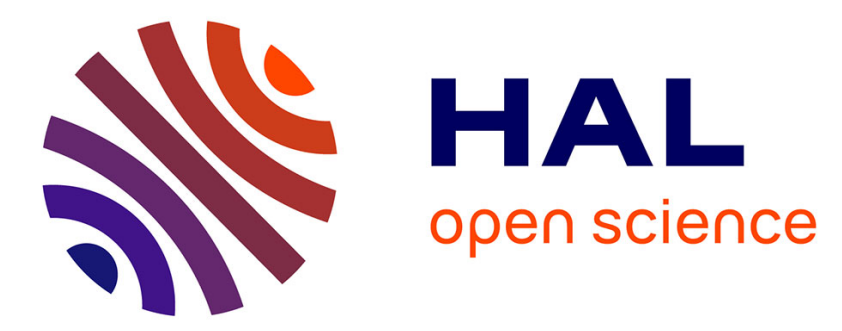

\title{
The emerging role of synaptic cell-adhesion pathways in the pathogenesis of autism spectrum disorders.
}

Catalina Betancur, Takeshi Sakurai, Joseph D Buxbaum

\section{To cite this version:}

Catalina Betancur, Takeshi Sakurai, Joseph D Buxbaum. The emerging role of synaptic cell-adhesion pathways in the pathogenesis of autism spectrum disorders.. Trends in Neurosciences, 2009, 32 (7), pp.402-412. 10.1016/j.tins.2009.04.003 . inserm-00401195

\section{HAL Id: inserm-00401195 https://www.hal.inserm.fr/inserm-00401195}

Submitted on 2 Jul 2009

HAL is a multi-disciplinary open access archive for the deposit and dissemination of scientific research documents, whether they are published or not. The documents may come from teaching and research institutions in France or abroad, or from public or private research centers.
L'archive ouverte pluridisciplinaire HAL, est destinée au dépôt et à la diffusion de documents scientifiques de niveau recherche, publiés ou non, émanant des établissements d'enseignement et de recherche français ou étrangers, des laboratoires publics ou privés. 


\title{
The emerging role of synaptic cell-adhesion pathways in the pathogenesis of autism spectrum disorders
}

\author{
Catalina Betancur ${ }^{1}$, Takeshi Sakurai ${ }^{2-6}$ and Joseph Buxbaum ${ }^{2,3,4,7,8}$ \\ ${ }^{1}$ INSERM U952, Université Pierre et Marie Curie, Paris 75005, France
}

${ }^{2}$ Laboratory of Molecular Neuropsychiatry, ${ }^{3}$ the Seaver Autism Center for Research and Treatment, ${ }^{4}$ Department of Psychiatry, ${ }^{5}$ Pharmacology and Systems Therapeutics, ${ }^{6}$ Black Family Stem Cell Institute, ${ }^{7}$ Department of Neuroscience, and ${ }^{8}$ Department of Genetics and Genomic Sciences, Mount Sinai School of Medicine, New York, New York 10029, USA

Correspondence should be addressed to: Dr. Joseph Buxbaum, Department of Psychiatry, Mount Sinai School of Medicine, One Gustave L. Levy Place, Box 1668, New York, New York 10029, tel: 212-6598862, fax: 212-828-4221, e-mail: joseph.buxbaum@mssm.edu

\begin{abstract}
Recent advances in genetics and genomics have unveiled numerous cases of autism spectrum disorders (ASDs) associated with rare, causal genetic variations. These findings support a novel view of ASDs in which many independent, individually rare genetic variants, each associated with a very high relative risk, together explain a large proportion of ASDs. Although these rare variants impact diverse pathways, there is accumulating evidence that synaptic pathways, including those involving synaptic cell adhesion, are disrupted in some subjects with ASD. These findings provide insights into the pathogenesis of ASDs and allow for the development of model systems with construct validity for specific causes of ASDs. In several neurodevelopmental disorders frequently associated with ASD, including fragile $X$ syndrome, Rett syndrome, and tuberous sclerosis, animal models have led to the development of new therapeutic approaches, giving rise to optimism with other causes of ASDs.
\end{abstract}




\section{Recent advances in the genetics of autism}

Autism and autism spectrum disorders (ASDs) are neurodevelopmental disorders with characteristic behavioral abnormalities, including deficits in reciprocal social interactions and communication, and the presence of repetitive behaviors and/or restricted interests. Onset occurs during the first three years of life and there is a male preponderance, with a sex ratio of $4: 1$. The incidence of ASDs is now estimated at 6 in $1000^{[1]}$. Family and twin studies have demonstrated that ASDs have a very high heritability. ASDs are etiologically heterogeneous, with an underlying genetic disorder identified in $10 \%-25 \%$ of cases, including monogenic disorders (e.g., fragile X syndrome [FXS], tuberous sclerosis, Rett syndrome), chromosomal aberrations and genomic imbalances ${ }^{[2]}$. It has been widely assumed that the remaining cases for which a causal factor has not been identified (referred to as "idiopathic" ASDs) are the result of interactions of multiple genetic variants of weak effect. This has led to the use of linkage and association studies to identify potential susceptibility loci of weak effect, few if any of which, however, have been consistently replicated ${ }^{[2]}$.

Recent technological developments, including advances in the detection of copy number variations (CNVs) (deletions and duplications) and rare point mutations have begun to alter the conceptualization of ASDs, as the results indicate that an ever increasing proportion of ASDs are due to rare, causal, single locus genetic changes that can be either inherited or de novo in origin. Recent findings point to the existence of many distinct autism loci, with de novo CNVs and mutations playing important roles (de novo CNVs have been identified in $10 \%$ of sporadic cases and in $2 \%-3 \%$ of families having two or more affected children ${ }^{[3,4]}$ ). The proportion of ASDs attributed to causal loci are likely to increase with the use of higher resolution microarrays and high-throughput sequencing.

This conceptual shift in ASDs can be summarized as a move from a 'common diseasecommon variant' perspective to a 'multiple rare variants' perspective (Box 1). The multiple rare variants model in ASD is reminiscent of the current conceptualization of intellectual disability (ID) ${ }^{[5,6]}$, in which defects in many different genes cause a similar behavioral phenotype. In fact, numerous genetic syndromes that cause ID can also present with ASD, suggesting the existence of common pathways in these two disorders. More broadly, variation in a given locus can lead to other neuropsychiatric disorders beyond ASDs and ID, suggesting that clinical diverse phenotypes may share similar genetic underpinnings.

Interestingly, many of the rare variants identified in ASDs relate to synaptic cell adhesion molecule (CAM) pathways, shedding light on the pathogenesis of ASDs and contributing to the identification of further candidate genes for ASDs. We review the genetic evidence for alterations in synaptic CAM pathways in ASDs and discuss how they might in turn result in cellular deficits based on studies on genetically modified mice and in vitro experiments. While these findings indicate that ASDs are much more heterogeneous in terms of etiology than previously thought, we note that the identification of any causal rare variant in ASDs has 
immediate clinical relevance for genetic counseling and that these rare variants are amenable to modeling in experimental systems.

\section{Synaptic cell adhesion molecules}

Within the synapse, a complex molecular interplay takes place between functional domains of the pre and postsynaptic elements, including the postsynaptic density, actin cytoskeleton, and membrane trafficking domains, supporting the formation and maintenance of synapses. Structural and functional alterations of synaptic connections, including abnormal density and morphology of dendritic spines, synapse loss, and aberrant synaptic signaling and plasticity, have all been suggested to be involved in psychiatric and neurological disorders ${ }^{[7]}$. Molecular components of synapses, including synaptic CAMs and scaffolding proteins, play crucial roles in the structure and function of synaptic connections.

CAMs are involved in the initial contact between pre- and post-synaptic cells, in maintaining synaptic adhesion, and as anchors for scaffolding proteins (Figure 1). These scaffolding proteins in turn assemble signaling molecules, neurotransmitter receptors, and proteins in the actin cytoskeleton, all working together in the development and plasticity of synapses ${ }^{[8-10]}$. Recent genetic studies support the involvement of genes coding for synaptic CAMs, scaffolding proteins, and downstream signaling molecules, in developmental delay and ASD, both as causal genes and as potential susceptibility genes (Table 1).

\section{Neuroligins, neurexins and SHANK3}

The best-characterized synaptic CAM pathways implicated in ASDs are those involving neuroligins and neurexins, pathways also supported by findings with the $\mathrm{SH} 3$ and multiple ankyrin repeat domains 3 (SHANK3) scaffolding protein. The evidence for rare variants in each of these genes will be summarized here, before discussing less well-characterized synaptic CAM pathways involved in ASDs.

\section{Neuroligins}

Neuroligins (NLGNs) are postsynaptic CAMs that support synapse formation of both excitatory and inhibitory synapses in vitro ${ }^{[11]}$. There are five $N L G N$ genes, including two $X-$ linked genes (NLGN3 and NLGN4X) and one Y-linked gene (NLGN4Y). A screen of the sexlinked NLGN genes in 158 ASD families identified a frameshift mutation in NLGN4X and a non-synonymous change in NLGN3 in two families having each two affected brothers, one with autism and the other with Asperger syndrome ${ }^{[12]}$, implicating for the first time these two genes in the etiology of ASDs. These mutations in NLGN3 and NLGN4X result in intracellular retention of the mutant proteins in the endoplasmic reticulum in vitro, and the reduced levels of proteins that reach the cell surface show decreased affinity to neurexin ${ }^{[13-15]}$. Furthermore, when overexpressed in hippocampal neurons, the ASD-associated mutations fail to support the formation of presynaptic terminals ${ }^{[13]}$. The introduction of the NLGN3 mutation into N/gn1 
drastically reduces spiny density and suppresses endogenous excitatory synapse function in cultured neurons ${ }^{[16]}$.

Subsequently, a mutation in NLGN4X resulting in a premature stop codon was identified in a multi-generational pedigree with 13 affected males having either non-specific intellectual disability (ID) (10 individuals), ID with ASD (2 individuals), or ASD without ID (1 individual) ${ }^{[17]}$. Mutation screening of NLGN3 and NLGN4X in independent ASD samples failed to identify other clear pathogenic mutations ${ }^{[18-22]}$, although several $N L G N 4 X$ missense variants ${ }^{[23]}$ and NLGN3 and NLGN4X splice isoforms ${ }^{[24]}$ of unknown clinical significance were identified. These results indicate that although mutations in NLGNs are not frequent causes of ASDs, disruption of NLGNs can lead to rare cases of ASDs and/or ID. Additional studies have found NLGN4X deletions associated with ASDs as well as with a wider variety of neuropsychiatric conditions (including Tourette syndrome, attention deficit-hyperactivity disorder, learning disorders, anxiety and depression) ${ }^{[4,25-28]}$ while other studies have shown that loss of $N L G N 4 X$ is not associated with a phenotype in certain cases ${ }^{[26,29]}$.

Some of these causal mutations in NLGNs have been directly modeled in mice. Nlgn3 R451C knock-in mice, which replicate the amino acid change found in a human NLGN3 mutation ${ }^{[12]}$, were reported to show impaired social interactions, enhanced spatial learning abilities, and enhanced inhibitory synaptic transmission in the somatosensory cortex with no apparent effect on excitatory synapses ${ }^{[30]}$. This study did not report an analogous phenotype in Nlgn3-knockout animals and the authors thus proposed that the R451C mutation may represent a gain-of-function mutation. However, the interpretation is complicated by a recent report that could not replicate the observed changes in independently-generated R451C knock-in mice, although there were differences in the genetic background ${ }^{\left[{ }^{31]}\right.}$. Recently, another group reported that their NIgn3 knockout mice show reduced ultrasound vocalizations

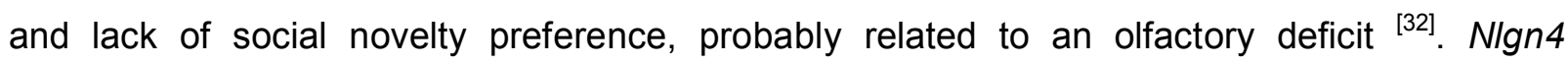
knockouts show selective deficits in reciprocal social interactions and communication that are reminiscent of ASDs ${ }^{[33]}$. Note, however, that in these and other studies with knockout mice, the relevance of models lacking two copies of a gene to a condition that is heterozygous in patients is questionable.

Whereas in vitro data suggest that neuroligins play a role in synapse formation ${ }^{[11]}$, studies with triple Nlgn1-Nlgn2-Nlgn3 knockout mice show that elimination of neuroligins does not affect synapse numbers in the brain but alters the recruitment of postsynaptic receptors to glutamatergic, $\gamma$-aminobutyric acid (GABA)ergic, and glycinergic synapses ${ }^{[34]}$. These findings indicate that neuroligins are essential for proper synapse maturation and function but not for the initial formation of synaptic contacts ${ }^{[35]}$. Based on comprehensive studies using both overexpression and genetically modified mice, it is becoming clear that neuroligins differentially specify synapse neurochemistry (excitatory or inhibitory), with different neuroligins acting on distinct types of synapses via activity-dependent mechanisms ${ }^{[16]}$. Thus, when Nlgn1 is overexpressed in cultured neurons, it increases excitatory but not inhibitory 
synaptic responses, whereas Nlgn2 overexpression selectively enhances inhibitory synapses

${ }^{[16]}$. Accordingly, NIgn1 knockout mice exhibit impaired N-methyl-D-aspartate (NMDA) receptor signaling, whereas NIgn2 knockout mice have reduced inhibitory synaptic transmission ${ }^{[16]}$. Notably, the synapse-boosting activity of Nlgn1 and Nlgn2 is suppressed by inhibition of synaptic activity, indicating that the effects of neuroligins are dependent on synaptic signaling [16].

\section{Neurexins}

Neurexins (NRXN1-NRXN3) are predominantly presynaptic CAMs and are binding partners of the postsynaptic neuroligins ${ }^{[36]}$. Neuroligins induce presynaptic differentiation in contacting axons ${ }^{[37]}$, a response mediated by neurexin ${ }^{[38]}$. Conversely, neurexin induces postsynaptic differentiation in glutamatergic synapses through interactions with $\operatorname{Nlgn} 1,3,4$ and in GABAergic synapses through interactions with Nlgn2 ${ }^{[39]}$. Although previously considered presynaptic molecules, neurexins are also expressed postsynaptically, where they block the synaptogenic activity of neuroligins by modulating the strength of neuroligin/neurexin interactions ${ }^{[40]}$. In addition, neurexins regulate postsynaptic NMDA receptor function through a cell-autonomous postsynaptic mechanism ${ }^{[41]}$.

There are three neurexin genes (NRXN1-NRXN3), each of which encodes two major variants, $\alpha$ (long) and $\beta$ (short), differing in their extracellular domains. CNV analysis using single nucleotide polymorphism (SNP) arrays in $\sim 1200$ families with at least two individuals with ASD identified a 2p16.3 deletion involving NRXN1 in two sisters ${ }^{[42]}$. Subsequent studies also identified rare $N R X N 1$ deletions $[4,43,44]$ and chromosomal abnormalities involving $2 \mathrm{p} 16.3 / N R X N 1$ in ASDs ${ }^{[45]}$. Rare sequence variants in NRXN1 have also been reported in ASD, but their clinical significance is unclear at present ${ }^{[45-47]}$. It should be noted however that both deletions and chromosomal aberrations disrupting NRXN1 have been reported in healthy carriers ${ }^{[45,48,49]}$, suggesting that heterozygous inactivation of $N R X N 1$ is not by itself sufficient to cause ASD and that other factors are required to produce the ASD phenotype. Furthermore, NRXN1 deletions have recently been shown to confer risk for schizophrenia, suggesting an etiological overlap between this disorder and ASD (Box 2).

Double and triple $\alpha$-Nrxn knockout mice show synaptic transmission defects without any obvious impairment in axon guidance or synapse formation [50, 51]. $\mathrm{Ca}^{2+}$-triggered neurotransmitter release is severely depressed in these animals due to altered functional coupling of $\mathrm{Ca}^{2+}$ channels to the presynaptic membrane, an effect specifically rescued by $\alpha$ Nrxn1 but not by $\beta-N r x n s{ }^{[52]}$. Interestingly, $C D K 5$ and $C A S K$, two intracellular mediators of NRXN/NLGN-induced synaptogenesis ${ }^{[53]}$, have been found to be either mutated or deleted in ID $^{[54-56]}$.

\section{SHANK3}

SHANK3 is a member of the SHANK synaptic scaffolding proteins that are abundant in the postsynaptic density ${ }^{[57-59]}$, where they interact with neuroligins directly ${ }^{[60]}$ or indirectly ${ }^{[61]}$. 
SHANK3 has multiple protein interaction domains, interfacing between glutamate receptor complexes and actin regulatory proteins, and is therefore well suited to play a role in spine morphogenesis and synaptic plasticity, functioning as a "master" scaffolding protein ${ }^{[58]}$. When overexpressed in cultured hippocampal neurons, Shank3 promotes the maturation and enlargement of dendritic spines ${ }^{[62]}$. Knock-down of Shank3 in hippocampal neurons decreases spiny density while transfection of Shank3 in aspiny neurons induces the formation of dendritic spines with functional synapses ${ }^{[63]}$.

The $22 q 13.3$ deletion syndrome is a microdeletion disorder characterized by global developmental delay, hypotonia, markedly delayed or absent speech, autistic behavior, and minor dysmorphic features ${ }^{[64]}$. Molecular characterization of individuals with the $22 q 13.3$ deletion syndrome identified a minimal deleted region of $100 \mathrm{~kb}$ containing three genes, SHANK3, ACR, and RAB2LB ${ }^{[65]}$. Evidence for the involvement of SHANK3 as the critical gene responsible for the ID and speech deficits associated with deletion of 22q13.3 first came from a patient with a balanced chromosomal translocation disrupting SHANK3 ${ }^{[66]}$. Further support for this gene came from a small number of individuals with minimal 22q13.3 deletions who were shown to have breakpoints situated within SHANK3, including several with a recurrent breakpoint within intron $8^{[67-69]}$. Morever, individuals with a ring chromosome 22 typically have deletions of the long arm of the chromosome and exhibit the 22q13 deletion phenotype, but subjects with an intact SHANK3 gene are phenotypically normal ${ }^{[70]}$.

Given the presence of autistic behavior in patients with 22q13.3 deletions [64, 69-71], variations in the SHANK3 gene were studied in 227 subjects with ASD using fluorescent in situ hybridization (FISH) and direct sequencing ${ }^{[68]}$. Alterations in SHANK3 were identified in three families: 1) a de novo 22q13 terminal deletion with the breakpoint in intron 8 of SHANK3 in a boy with autism and ID; 2) a nucleotide insertion creating a frameshift mutation in two affected brothers, likely resulting from germinal mosaicism in the mother; and 3) a terminal $22 q 13$ deletion in a girl with autism and a reciprocal duplication in her brother with Asperger syndrome, both resulting from a paternal chromosomal translocation ${ }^{[68]}$. This study confirmed that haploinsufficiency of SHANK3 accounts for the 22q13 deletion phenotype. In addition, the ASD phenotype associated with both loss and gain of SHANK3 supports a critical role for SHANK3 gene dosage in neurodevelopment. The SHANK3 frameshift mutation (3680insG) results in a truncated protein lacking crucial protein-protein interaction domains (Homerbinding, cortactin-binding, Abp1-binding, and SAM domains) involved in glutamate receptor and actin binding, spine formation and maturation and synaptic targeting. Accordingly, no synaptic localization was observed after overexpression of the mutant protein in rat hippocampal neurons ${ }^{[68]}$.

Two recent studies confirmed the role of SHANK3 in ASDs, identifying one de novo mutation and two deletions in 400 subjects ${ }^{[72]}$ and one de novo splice site mutation in 427 subjects ${ }^{[73]}$ (note that the latter study did not screen for deletions). These findings, together 
with those reported initially ${ }^{[68]}$ lead to an estimated prevalence of at least $0.47 \%(7 / 1054)$ SHANK3 pathogenic variants in ASDs.

Knockout mice of the related molecule Shank1 show smaller dendritic spines of CA1 pyramidal neurons and weaker synaptic transmission, along with altered learning and memory ${ }^{[74]}$. Our own preliminary studies with heterozygous Shank3-knockout mice support a clear role for SHANK3 in synaptic transmission and plasticity, as evidenced by deficits in hippocampal long-term potentiation (T. Sakurai, J.D. Buxbaum et al., unpublished).

\section{Other synaptic CAMs and scaffolding proteins associated with ASD and ID}

The cadherin $(\mathrm{CDH})$ and protocadherin $(\mathrm{PCDH})$ families include a large number of proteins that are typical synaptic CAMs whose differential expressions may be important for specific synaptic formations ${ }^{[75,76]}$. This family of proteins usually undergoes homophilic interactions to support cell adhesion. N-cadherin ( $\mathrm{CDH} 2)$, a prototypical cadherin, has been shown to be associated with both excitatory and inhibitory synapses in cultured hippocampal neurons ${ }^{[77]}$. Protocadherins have also been shown to be present at synaptic sites, where they may contribute to synaptic specificity in concert with classical cadherins ${ }^{[78,79]}$.

A recent study identified CNVs involving the $P C D H 9$ gene and a de novo translocation deleting the $C D H 18$ gene in ASDs ${ }^{[4]}$. Moreover, in a study of consanguineous families with children with ASD, homozygous deletions implicated $P C D H 10$, whose expression is regulated by neuronal activity, in ASDs ${ }^{[80]}$. Epilepsy, female-restricted, with mental retardation (EFMR) is a disorder with an unusual mode of inheritance, affecting females. Very recently, mutations in the X-linked PCDH19 gene were identified in 7 families with EFMR, some leading to

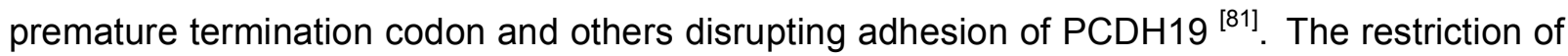
EFMR to females has been suggested to be due to $X$ inactivation of the gene, with hemizygous males having a homogeneous population of PCDH19-negative cells and affected females being mosaics for PCDH19-negative and wild-type cells. As the PCDH19 cell adhesion is possibly homophilic, this mosaicism might disrupt cell-cell communication more severely than a simple loss of PCDH19.

Two other genes encoding CAMs of the cadherin and immunoglobulin (Ig) superfamilies, CDH15 and KIRREL3, were recently shown to be disrupted by a chromosomal translocation in a patient with ID ${ }^{[82]}$. Subsequent mutation screening in a cohort of patients with ID identified several rare missense variants in both genes ${ }^{[82]}$. The $\mathrm{CDH} 15$ variants alter the cell-cell adhesion properties of the protein in vitro, while KIRREL3 was shown to interact in neurons with the synaptic scaffolding protein CASK, recently implicated in X-linked ID ${ }^{[56]}$.

One recent discovery in ASD is the presence of recurrent $16 \mathrm{p} 11.2$ microdeletions and microduplications in as much as $1 \%$ of cases ${ }^{[4,44,83,84]}$. The critical interval, which is flanked by low copy repeats, contains $\sim 30$ genes, including TAOK2 and MAPK3, which are part of a signaling pathway directly linked to the synaptic CAMs $\mathrm{N}$-cadherin and $\mathrm{PCDH} 8{ }^{[85]}$. $\mathrm{PCDH} 8$ cell surface expression is modulated by neuronal activity via a mechanism that involves 
activation of the kinases TAOK2 and MAPK3, which in turn leads to the internalization of PCDH8 and subsequent weakening of synapses. It would be interesting to determine if specific disruption of TAOK2 and/or MAPK3 is associated with ASD.

The Ig superfamily of CAMs is a large family of CAMs that are classified into subfamilies based on their structure. They interact both homophillically and heterophillically to support neuronal cell adhesion ${ }^{[86]}$. The L1 CAM (L1CAM), a member of the L1 subfamily, is an important mediator of neuronal migration, process outgrowth and synaptic targeting ${ }^{[87]}$, and was the first CAM to be associated with a genetic disorder. Mutations in the L1CAM gene are responsible for a syndromic form of X-linked ID ${ }^{[88]}$.

The contactins (CNTNs 1-6) are glycosyl phosphatidylinositol-linked Ig superfamily CAMs, which have been shown to be involved in axon growth, guidance, and synapse formation and plasticity ${ }^{[89]}$. Disruption of CNTN4 (3p26.3) results in 3p deletion syndrome, which includes ID, growth retardation and dysmorphic features, and can be associated with ASD ${ }^{[90]}$. Array-based comparative genomic hybridization identified paternally-inherited CNVs in the CNTN4 gene in ASDs, including a deletion in 2 siblings and a duplication in an unrelated individual ${ }^{\text {[91] }}$ Because the parents were reportedly healthy, these findings suggest either incomplete penetrance or a polymorphic (i.e. neutral) variant. More recently, compelling evidence for a role for CNTN4 deletions and duplications in ASDs was observed in a CNV analysis involving over 2000 cases ${ }^{[44]}$; here too the CNVs were most commonly inherited. In addition, small deletions close to CNTN3 (3p12.3) have been identified in ASDs ${ }^{[80]}$. Since studies in rodents show that both CNTN3 and CNTN4 are highly expressed in the developing brain at the time of synaptogenesis ${ }^{\left[{ }^{92}\right]}$, these contactins may be associated with ASDs and ID through alterations in synapse formation and function.

The contactin associated proteins (CNTNAP1-5) show structural similarity to neurexin, even though they belong to different families. It is not yet known if CNTNAPs have a synaptic function like contactins or neurexins ${ }^{[93]}$, although CNTNAP2 has been shown to be present in the synaptic plasma membrane fraction of brain lysates ${ }^{[94]}$. CNTNAP2 interacts with CNTN2 and localizes at the juxtaparanode of myelinated nerves ${ }^{[95,96]}$. Moreover, CNTNAP2 is enriched in language-related circuits involving the frontal and anterior temporal lobes, striatum, and dorsal thalamus ${ }^{[97]}$. Homozygous CNTNAP2 mutations were described in Amish children with cortical dysplasia-focal epilepsy syndrome, characterized by neuronal migration abnormalities, seizures, ID and ASD ${ }^{[98]}$. A de novo chromosomal inversion disrupting AUTS2 and CNTNAP2 was recently described in a child with cognitive and social delay and autistic traits ${ }^{[94]}$. Because polymorphic CNVs of the AUTS2 locus had been described in healthy individuals, the authors focused on CNTNAP2, sequencing the gene in 635 patients with ASD and 942 controls. Among patients, they identified a total of 27 nonsynonymous changes; 13 were rare and unique to patients and 8 of these were predicted to be deleterious by bioinformatic approaches and/or altered residues conserved across all species. Overall, this resequencing data demonstrated a moderate increase in the burden of rare variants in cases 
versus controls, suggesting that the contribution of rare variants of CNTNAP2 may be modest in ASD ${ }^{[94]}$. However, the role of CNTNAP2 in ASD received support by the recent report of a CNTNAP2 deletion in a female with autism ${ }^{[99]}$. Consistent with a variable expressivity associated with many genetic and genomic variants, CNTNAP2 was also reported to be disrupted by a chromosomal aberration in a family with Gilles de la Tourette syndrome and obsessive compulsive disorder ${ }^{[100]}$ (and see Box 2).

As more rare causal variants are identified, we will gain further insight into CAMs and CAM-signaling pathways in ID and ASD. For instance, the recently described 3q29 microdeletion ID syndrome ${ }^{[101]}$ includes PAK2 (a synaptic signaling molecule) and DLG1 (a synaptic scaffolding protein), which are homologous to two X-linked ID genes, PAK3 and DLG3. These synaptic scaffolding proteins appear to bind to neuroligin ${ }^{[60]}$ as well as to nectins, which are Ig superfamily CAMs ${ }^{[102]}$ involved in synapse formation ${ }^{[103]}$.

\section{Common variants in CAMs and susceptibility to ASDs}

Although this review focuses on the most recent discoveries of rare variants in ASDs, there remains empirical support for a role for common variants in the disorder, most notably because of the presence of a subtle phenotype in a large proportion of family members, the so-called broad autism phenotype ${ }^{[104]}$, more commonly observed in multiply affected families ${ }^{[105]}$. Association studies of some synaptic CAMs have suggested that common variants in such genes might represent susceptibility loci. Positive, replicated evidence for association of ASDs with common variants in CAMs has been described for CNTNAP2 ${ }^{[97,106]}$, NRCAM ${ }^{[107-}$ ${ }^{109]}$, and reelin ${ }^{[110]}$. However, recent genome-wide association analyses in considerably larger samples do not provide support for a strong association between ASDs and these genes. In contrast, genome-wide association analysis across multiple samples, totaling over 2600 ASD subjects, has identified six SNPs that lie between the cadherin 9 (CDH9) and cadherin 10 $(C D H 10)$ genes on 5 p14.1 as associated with ASDs with an important population attributable risk ${ }^{[111]}$, suggesting that common variants in certain neuronal CAMs can increase risk for ASDs.

\section{CAMs and ASDs: emerging pathogenetic mechanisms}

Neuropathological and imaging studies in autism have failed to identify consistent abnormalities of brain structure, except for brain overgrowth in about $20 \%$ of cases. Similarly, there is not as yet an accepted microscopic neuropathology in autism, although only a few postmortem studies have been performed. The genetic studies reviewed here implicate synaptic CAM pathways as etiological factors in ASD, but the nature of the underlying pathogenetic mechanisms and how they lead to the development of the social and communication deficits in individuals with ASDs remains largely unknown. Nevertheless, the results obtained in mutant mice as well as in vitro experiments provide a glimpse of some of the cellular events that might be involved in the development of ASD, including altered 
synaptic maturation, connectivity and stabilization. The in vitro data with the scaffolding protein SHANK3 as well as in vivo data in Shank1-knockout mice depicting dramatic alterations in spine morphology ${ }^{[62,63,74]}$, represent good examples of how altered expression of such genes can disrupt critical synaptic function. Similarly, the importance of the transsynaptic neuroligin-neurexin complex for proper synaptic function is clear from the marked

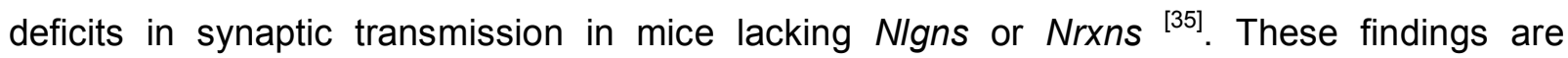
particularly interesting in view of previous studies showing abnormal development of dendritic spines in various neurodevelopmental disorders associated with ASD, including fragile $X$ syndrome, Rett syndrome, Angelman syndrome, tuberous sclerosis, Down syndrome, and William's syndrome ${ }^{[112,113]}$. In addition, the onset of autism occurs before the age of three years, during a critical period of synapse formation and maturation, and this time course is consistent with a role of synaptic dysfunction in the pathogenesis of autism. Recent studies showing that neuroligins validate transient synapses in an activity dependent manner ${ }^{[16]}$ also support the hypothesis that ASD might result from disruption of postnatal experiencedependent synaptic plasticity ${ }^{[114]}$.

The observation of epilepsy in about a third of patients with autism has prompted speculation that ASDs may be due to an enhanced excitation/inhibition (E/I) ratio resulting from aberrant formation and function of excitatory versus inhibitory cortical synapses ${ }^{[115]}$. Genetic variants that disrupt SHANK3, NLGN3, NLGN4X or NRXN1 genes implicate alterations in glutamatergic synapse development. However, NRXN/NLGN interactions are also involved in inhibitory synapse development in ASD ${ }^{[11,39,116]}$, and therefore could also play a role in ASD and ID through disruption of inhibitory synapses. In vivo studies with Shank1 knockout mice show reduced excitatory synaptic strength ${ }^{[74]}$, something we also observe in Shank3 heterozygotes (T. Sakurai, J.D. Buxbaum et al., unpublished), so we must also consider decreased E/I ratios in a subset of ASDs. Brain imaging studies suggest that the core features of autism could be related to disturbed connectivity, both interhemispheric and intracortical ${ }^{[117]}$. It will be interesting to determine whether disruption of the CAM pathways leads to alterations in E/l balance, creating a noisy or muted system and contributing to altered connectivity. Certainly, the elegant neurobiological investigations on neuroligins and neurexins illustrate how mutations in these genes might alter the balance between excitatory and inhibitory neurotransmission ${ }^{[35]}$, ultimately disrupting the cortical and subcortical brain networks involved in social cognition and language.

There is, of course, still a huge amount of work to be done to understand the role of synaptic CAMs and related proteins in the pathophysiology of ASDs. The identification of highly penetrant mutations in these genes is just a first step, providing molecular targets for neurobiological studies to unravel the complex pathogenesis of ASDs. 


\section{Novel therapeutic approaches in ASDs}

FXS, which is the most common inherited cause of cognitive impairment, is found in about $2 \%$ of individuals with ASD ${ }^{[118]}$, and, conversely, $\sim 50 \%$ of individuals with FXS have autism or ASD ${ }^{[119]}$. FXS is caused by pathological trinucleotide expansions in the FMR1 gene, which codes for the Fragile $X$ mental retardation protein (FMRP). FMRP functions as a repressor of mRNA translation and participates in the transport of mRNA species that play a role in synaptic plasticity in an activity-dependent manner ${ }^{[120]}$. The reduction of FMRP expression in FXS leads to enhanced synaptic protein synthesis after stimulation of metabotropic glutamate receptors (mGluR) ${ }^{[121]}$. Remarkably, blocking the function of mGluR by either genetic means or by pharmacological agents reverses some of the synaptic abnormalities and higher-order phenotypes observed in model systems of FXS, including those in mice, flies, and worms ${ }^{[122-}$ ${ }^{125]}$. This hypothesis has led to a clinical trial currently underway of an mGluR5 reverse agonist in FXS. Recent studies of mouse models of other genetic disorders associated with autism have also shown striking and dramatic reversal of neurological defects. Rett syndrome, caused by mutations in the X-linked MECP2 gene, a transcriptional repressor, is considered a disorder of synapse development. Abnormal phenotypes in Mecp2-deficient mice can be rescued by reactivation of MeCP2 expression after birth ${ }^{[126,127]}$ or by administration of insulingrowth factor, which strongly promotes synaptic maturation ${ }^{[128]}$. Similarly, in Tsc1 or Tsc2 knockout mice (models of tuberous sclerosis), and Pten-knockout mice (which model the macrocephaly and behavioral abnormalities in ASD patients with PTEN mutations), the neurological abnormalities can be reversed by pharmacological inhibition of the mammalian target of rapamycin (mTOR) pathway ${ }^{[129-132]}$. Rapamycin improved survival, reduced seizures, and reversed cognitive abnormalities and associated cellular deficits, including neuronal hypertrophy and altered hippocampal synaptic plasticity. A multi-site clinical trial of rapamycin in individuals with tuberous sclerosis is ongoing, and interim reports have been positive for short-term memory effects. Smaller scale studies with other etiological subgroups are also underway, including intranasal insulin in children with 22q13 deletion syndrome ${ }^{[133]}$.

The surprising phenotypic reversibility observed in these animal models after birth -or even in the adult- suggests that these "developmental" disorders could at least in part be due to the ongoing lack of functional protein and/or that the brain remains extremely plastic even after an impaired neurodevelopmental history. The importance of the results summarized here cannot be overstated as they imply that identifying causal forms of ASDs and ID might lead to successful targeted therapies for these disorders, some of which possibly being specific for a subtype of the disorders ("personalized medicine").

\section{Concluding remarks}

During the past few years, researchers have identified several causal genes for ASD and ID encoding synaptic CAMs and associated molecules involved in synaptic function. We should note that many of these findings will need to be replicated in additional, large samples. In 
addition, the functional relevance of some of the variants needs to be critically examined. In parallel, as causal genes for ASD and ID are identified and validated in genetic studies, we will need to clarify how these genes cause the ASD and/or ID phenotype, making use of in vitro and in vivo model systems. Causal variants are readily studied in model systems as the technology to introduce or disrupt genes in cellular and animal models is widespread. The understanding of these pathways in neurodevelopmental disorders will likely lead to novel, targeted therapeutic strategies, with the recent developments in experimental treatments in fragile $X$ syndrome and other ASD-associated disorders representing important paradigms.

\section{Acknowledgements}

Work in the authors' laboratories is supported by the Seaver Foundation, Handler Foundation, National Institutes of Health, Autism Speaks, Simons Foundation, Stanley Foundation, INSERM, Fondation de France, Fondation pour la Recherche Médicale and Fondation Orange. JDB is the G. Harold and Leila Y. Mathers Professor at Mt. Sinai School of Medicine. The authors declare no competing financial interests.

\section{References}

1 Fombonne, E. (2005) Epidemiology of autistic disorder and other pervasive developmental disorders. J Clin Psychiatry 66 Suppl 10, 3-8

2 Abrahams, B.S. and Geschwind, D.H. (2008) Advances in autism genetics: on the threshold of a new neurobiology. Nat Rev Genet 9, 341-355

3 Sebat, J. et al. (2007) Strong association of de novo copy number mutations with autism. Science 316, 445-449

4 Marshall, C.R. et al. (2008) Structural variation of chromosomes in autism spectrum disorder. Am J Hum Genet 82, 477-488

5 Chelly, J. et al. (2006) Genetics and pathophysiology of mental retardation. Eur J Hum Genet 14, 701-713

6 Chiurazzi, P. et al. (2008) XLMR genes: update 2007. Eur J Hum Genet 16, 422-434

7 Blanpied, T.A. and Ehlers, M.D. (2004) Microanatomy of dendritic spines: emerging principles of synaptic pathology in psychiatric and neurological disease. Biol Psychiatry 55, 1121-1127

8 Scheiffele, P. (2003) Cell-cell signaling during synapse formation in the CNS. Annu Rev Neurosci 26, 485-508

9 Gerrow, K. and El-Husseini, A. (2006) Cell adhesion molecules at the synapse. Front Biosci 11, 2400-2419

10 Okabe, S. (2007) Molecular anatomy of the postsynaptic density. Mol Cell Neurosci 34, 503-518

11 Chih, B. et al. (2005) Control of excitatory and inhibitory synapse formation by neuroligins. Science 307, 1324-1328

12 Jamain, S. et al. (2003) Mutations of the X-linked genes encoding neuroligins NLGN3 and NLGN4 are associated with autism. Nat Genet 34, 27-29

13 Chih, B. et al. (2004) Disorder-associated mutations lead to functional inactivation of neuroligins. Hum Mol Genet 13, 1471-1477

14 Comoletti, D. et al. (2004) The Arg451Cys-neuroligin-3 mutation associated with autism reveals a defect in protein processing. $J$ Neurosci $24,4889-4893$ 
15 Chubykin, A.A. et al. (2005) Dissection of synapse induction by neuroligins: effect of a neuroligin mutation associated with autism. $J$ Biol Chem 280, 22365-22374

16 Chubykin, A.A. et al. (2007) Activity-dependent validation of excitatory versus inhibitory synapses by neuroligin-1 versus neuroligin-2. Neuron $54,919-931$

17 Laumonnier, F. et al. (2004) X-linked mental retardation and autism are associated with a mutation in the NLGN4 gene, a member of the neuroligin family. Am J Hum Genet 74, 552-557

18 Vincent, J.B. et al. (2004) Mutation screening of X-chromosomal neuroligin genes: no mutations in 196 autism probands. Am J Med Genet B Neuropsychiatr Genet 129B, 82-84

19 Gauthier, J. et al. (2005) NLGN3/NLGN4 gene mutations are not responsible for autism in the Quebec population. Am J Med Genet B Neuropsychiatr Genet 132B, 74-75

20 Ylisaukko-oja, T. et al. (2005) Analysis of four neuroligin genes as candidates for autism. Eur $\mathrm{J}$ Hum Genet 13, 1285-1292

21 Blasi, F. et al. (2006) Absence of coding mutations in the X-linked genes neuroligin 3 and neuroligin 4 in individuals with autism from the IMGSAC collection. Am J Med Genet B Neuropsychiatr Genet 141B, 220-221

22 Wermter, A.K. et al. (2008) No evidence for involvement of genetic variants in the X-linked neuroligin genes NLGN3 and NLGN4X in probands with autism spectrum disorder on high functioning level. Am J Med Genet B Neuropsychiatr Genet 147B, 535-537

23 Yan, J. et al. (2005) Analysis of the neuroligin 3 and 4 genes in autism and other neuropsychiatric patients. Mol Psychiatry 10, 329-332

24 Talebizadeh, Z. et al. (2006) Novel splice isoforms for NLGN3 and NLGN4 with possible implications in autism. J Med Genet 43, e21

25 Chocholska, S. et al. (2006) Molecular cytogenetic analysis of a familial interstitial deletion Xp22.2-22.3 with a highly variable phenotype in female carriers. Am J Med Genet A 140, 604-610

26 Macarov, M. et al. (2007) Deletions of VCX-A and NLGN4: a variable phenotype including normal intellect. J Intellect Disabil Res 51, 329-333

27 Lawson-Yuen, A. et al. (2008) Familial deletion within NLGN4 associated with autism and Tourette syndrome. Eur J Hum Genet 16, 614-618

28 Kent, L. et al. (2008) X-linked ichthyosis (steroid sulfatase deficiency) is associated with increased risk of attention deficit hyperactivity disorder, autism and social communication deficits. $J$ Med Genet 45, 519-524

29 Mochel, F. et al. (2008) Normal intelligence and social interactions in a male patient despite the deletion of NLGN4X and the VCX genes. Eur J Med Genet 51, 68-73

30 Tabuchi, K. et al. (2007) A neuroligin-3 mutation implicated in autism increases inhibitory synaptic transmission in mice. Science 318, 71-76

31 Chadman, K.K. et al. (2008) Minimal aberrant behavioral phenotypes of neuroligin-3 R451C knock-in mice. Autism Research 1, 147-158

32 Radyushkin, K. et al. (2009) Neuroligin-3 deficient mice: Model of a monogenic heritable form of autism with an olfactory deficit. Genes Brain Behav [Epub ahead of print]

33 Jamain, S. et al. (2008) Reduced social interaction and ultrasonic communication in a mouse model of monogenic heritable autism. Proc Natl Acad Sci U S A 105, 1710-1715

34 Varoqueaux, F. et al. (2006) Neuroligins determine synapse maturation and function. Neuron 51, 741-754

35 Sudhof, T.C. (2008) Neuroligins and neurexins link synaptic function to cognitive disease. Nature 455, 903-911

36 Ichtchenko, K. et al. (1995) Neuroligin 1: a splice site-specific ligand for beta-neurexins. Cell 81, 435-443

37 Scheiffele, P. et al. (2000) Neuroligin expressed in nonneuronal cells triggers presynaptic development in contacting axons. Cell 101, 657-669 
38 Dean, C. et al. (2003) Neurexin mediates the assembly of presynaptic terminals. Nat Neurosci 6, 708-716

39 Graf, E.R. et al. (2004) Neurexins induce differentiation of GABA and glutamate postsynaptic specializations via neuroligins. Cell 119, 1013-1026

40 Taniguchi, H. et al. (2007) Silencing of neuroligin function by postsynaptic neurexins. J Neurosci 27, 2815-2824

41 Kattenstroth, G. et al. (2004) Postsynaptic N-methyl-D-aspartate receptor function requires alphaneurexins. Proc Natl Acad Sci U S A 101, 2607-2612

42 Autism Genome Project Consortium (2007) Mapping autism risk loci using genetic linkage and chromosomal rearrangements. Nat Genet 39, 319-328

43 Zahir, F.R. et al. (2008) A patient with vertebral, cognitive and behavioural abnormalities and a de novo deletion of NRXN1alpha. $J$ Med Genet 45, 239-243

44 Glessner, J.T. et al. Autism genome-wide copy number variation identifies ubiquitin and neuronal genes. Nature 459, 569-573

45 Kim, H.G. et al. (2008) Disruption of neurexin 1 associated with autism spectrum disorder. Am J Hum Genet 82, 199-207

46 Feng, J. et al. (2006) High frequency of neurexin 1 beta signal peptide structural variants in patients with autism. Neurosci Lett 409, 10-13

47 Yan, J. et al. (2008) Neurexin 1alpha structural variants associated with autism. Neurosci Lett 438, 368-370

48 Redon, R. et al. (2006) Global variation in copy number in the human genome. Nature 444, 444454

49 Rujescu, D. et al. (2009) Disruption of the neurexin 1 gene is associated with schizophrenia. Hum Mol Genet 18, 988-996

50 Missler, M. et al. (2003) Alpha-neurexins couple Ca2+ channels to synaptic vesicle exocytosis. Nature 423, 939-948

51 Dudanova, I. et al. (2007) Deletion of alpha-neurexins does not cause a major impairment of axonal pathfinding or synapse formation. J Comp Neurol 502, 261-274

52 Zhang, W. et al. (2005) Extracellular domains of alpha-neurexins participate in regulating synaptic transmission by selectively affecting $\mathrm{N}$ - and P/Q-type Ca2+ channels. J Neurosci 25, 4330-4342

53 Samuels, B.A. et al. (2007) Cdk5 promotes synaptogenesis by regulating the subcellular distribution of the MAGUK family member CASK. Neuron 56, 823-837

54 Venturin, M. et al. (2006) Mutations and novel polymorphisms in coding regions and UTRs of CDK5R1 and OMG genes in patients with non-syndromic mental retardation. Neurogenetics 7 , 59-66

55 Froyen, G. et al. (2007) Detection of genomic copy number changes in patients with idiopathic mental retardation by high-resolution $\mathrm{X}$-array-CGH: important role for increased gene dosage of XLMR genes. Hum Mutat 28, 1034-1042

56 Najm, J. et al. (2008) Mutations of CASK cause an X-linked brain malformation phenotype with microcephaly and hypoplasia of the brainstem and cerebellum. Nat Genet 40, 1065-1067

57 Sugiyama, Y. et al. (2005) Determination of absolute protein numbers in single synapses by a GFP-based calibration technique. Nat Methods 2, 677-684

58 Boeckers, T.M. (2006) The postsynaptic density. Cell Tissue Res 326, 409-422

59 Sheng, M. and Hoogenraad, C.C. (2007) The postsynaptic architecture of excitatory synapses: a more quantitative view. Annu Rev Biochem 76, 823-847

60 Meyer, G. et al. (2004) The complexity of PDZ domain-mediated interactions at glutamatergic synapses: a case study on neuroligin. Neuropharmacology 47, 724-733

61 Gerrow, K. et al. (2006) A preformed complex of postsynaptic proteins is involved in excitatory synapse development. Neuron 49, 547-562 
62 Sala, C. et al. (2001) Regulation of dendritic spine morphology and synaptic function by Shank and Homer. Neuron 31, 115-130

63 Roussignol, G. et al. (2005) Shank expression is sufficient to induce functional dendritic spine synapses in aspiny neurons. J Neurosci $25,3560-3570$

64 Cusmano-Ozog, K. et al. (2007) 22q13.3 deletion syndrome: a recognizable malformation syndrome associated with marked speech and language delay. Am J Med Genet C Semin Med Genet 145C, 393-398

65 Wilson, H.L. et al. (2003) Molecular characterisation of the 22q13 deletion syndrome supports the role of haploinsufficiency of SHANK3/PROSAP2 in the major neurological symptoms. $J$ Med Genet 40, 575-584

66 Bonaglia, M.C. et al. (2001) Disruption of the ProSAP2 gene in a $t(12 ; 22)(q 24.1 ; q 13.3)$ is associated with the 22q13.3 deletion syndrome. Am J Hum Genet 69, 261-268

67 Bonaglia, M.C. et al. (2006) Identification of a recurrent breakpoint within the SHANK3 gene in the 22q13.3 deletion syndrome. J Med Genet 43, 822-828

68 Durand, C.M. et al. (2007) Mutations in the gene encoding the synaptic scaffolding protein SHANK3 are associated with autism spectrum disorders. Nat Genet 39, 25-27

69 Philippe, A. et al. (2008) Neurobehavioral profile and brain imaging study of the 22q13.3 deletion syndrome in childhood. Pediatrics 122, e376-382

70 Jeffries, A.R. et al. (2005) Molecular and phenotypic characterization of ring chromosome 22. Am J Med Genet A 137, 139-147

71 Manning, M.A. et al. (2004) Terminal 22q deletion syndrome: a newly recognized cause of speech and language disability in the autism spectrum. Pediatrics 114, 451-457

72 Moessner, R. et al. (2007) Contribution of SHANK3 mutations to autism spectrum disorder. Am J Hum Genet 81, 1289-1297

73 Gauthier, J. et al. (2009) Novel de novo SHANK3 mutation in autistic patients. Am J Med Genet B Neuropsychiatr Genet 150B, 421-424

74 Hung, A.Y. et al. (2008) Smaller dendritic spines, weaker synaptic transmission, but enhanced spatial learning in mice lacking Shank1. J Neurosci 28, 1697-1708

75 Redies, C. et al. (2003) Cadherins as regulators for the emergence of neural nets from embryonic divisions. J Physiol Paris 97, 5-15

76 Arikkath, J. and Reichardt, L.F. (2008) Cadherins and catenins at synapses: roles in synaptogenesis and synaptic plasticity. Trends Neurosci 31, 487-494

77 Benson, D.L. and Tanaka, H. (1998) N-cadherin redistribution during synaptogenesis in hippocampal neurons. J Neurosci 18, 6892-6904

78 Phillips, G.R. et al. (2003) Gamma-protocadherins are targeted to subsets of synapses and intracellular organelles in neurons. J Neurosci 23, 5096-5104

79 Weiner, J.A. et al. (2005) Gamma protocadherins are required for synaptic development in the spinal cord. Proc Natl Acad Sci U S A 102, 8-14

80 Morrow, E.M. et al. (2008) Identifying autism loci and genes by tracing recent shared ancestry. Science 321, 218-223

81 Dibbens, L.M. et al. (2008) X-linked protocadherin 19 mutations cause female-limited epilepsy and cognitive impairment. Nat Genet 40, 776-781

82 Bhalla, K. et al. (2008) Alterations in CDH15 and KIRREL3 in patients with mild to severe intellectual disability. Am J Hum Genet 83, 703-713

83 Weiss, L.A. et al. (2008) Association between microdeletion and microduplication at 16p11.2 and autism. N Engl J Med 358, 667-675

84 Kumar, R.A. et al. (2008) Recurrent 16p11.2 microdeletions in autism. Hum Mol Genet 17, 628638

85 Yasuda, S. et al. (2007) Activity-induced protocadherin arcadlin regulates dendritic spine number by triggering $\mathrm{N}$-cadherin endocytosis via TAO2beta and p38 MAP kinases. Neuron $56,456-471$ 
86 Maness, P.F. and Schachner, M. (2007) Neural recognition molecules of the immunoglobulin superfamily: signaling transducers of axon guidance and neuronal migration. Nat Neurosci 10, 1926

87 Schmid, R.S. and Maness, P.F. (2008) L1 and NCAM adhesion molecules as signaling coreceptors in neuronal migration and process outgrowth. Curr Opin Neurobiol 18, 245-250

88 Kenwrick, S. et al. (2000) Neural cell recognition molecule L1: relating biological complexity to human disease mutations. Hum Mol Genet 9, 879-886

89 Karagogeos, D. (2003) Neural GPI-anchored cell adhesion molecules. Front Biosci 8, s1304-1320

90 Fernandez, T. et al. (2004) Disruption of contactin 4 (CNTN4) results in developmental delay and other features of 3p deletion syndrome. Am J Hum Genet 74, 1286-1293; addendum Am J Hum Genet (2008) 1282, 1385

91 Roohi, J. et al. (2009) Disruption of contactin 4 in 3 subjects with autism spectrum disorder. J Med Genet 46, 176-182

92 Yoshihara, Y. et al. (1995) Overlapping and differential expression of BIG-2, BIG-1, TAG-1, and F3: four members of an axon-associated cell adhesion molecule subgroup of the immunoglobulin superfamily. $J$ Neurobiol 28, 51-69

93 Pillai, A.M. et al. (2007) No effect of genetic deletion of contactin-associated protein (CASPR) on axonal orientation and synaptic plasticity. J Neurosci Res 85, 2318-2331

94 Bakkaloglu, B. et al. (2008) Molecular cytogenetic analysis and resequencing of contactin associated protein-like 2 in autism spectrum disorders. Am J Hum Genet 82, 165-173

95 Poliak, S. et al. (2003) Juxtaparanodal clustering of Shaker-like K+ channels in myelinated axons depends on Caspr2 and TAG-1. J Cell Biol 162, 1149-1160

96 Traka, M. et al. (2003) Association of TAG-1 with Caspr2 is essential for the molecular organization of juxtaparanodal regions of myelinated fibers. J Cell Biol 162, 1161-1172

97 Alarcon, M. et al. (2008) Linkage, association, and gene-expression analyses identify CNTNAP2 as an autism-susceptibility gene. Am J Hum Genet 82, 150-159

98 Strauss, K.A. et al. (2006) Recessive symptomatic focal epilepsy and mutant contactin-associated protein-like 2. N Engl J Med 354, 1370-1377

99 Rossi, E. et al. (2008) A 12Mb deletion at 7q33-q35 associated with autism spectrum disorders and primary amenorrhea. Eur J Med Genet 51, 631-638

100 Verkerk, A.J. et al. (2003) CNTNAP2 is disrupted in a family with Gilles de la Tourette syndrome and obsessive compulsive disorder. Genomics 82, 1-9

101 Willatt, L. et al. (2005) 3q29 microdeletion syndrome: clinical and molecular characterization of a new syndrome. Am J Hum Genet 77, 154-160

102 Kakunaga, S. et al. (2005) Nectin-like molecule-1/TSLL1/SynCAM3: a neural tissue-specific immunoglobulin-like cell-cell adhesion molecule localizing at non-junctional contact sites of presynaptic nerve terminals, axons and glia cell processes. J Cell Sci 118, 1267-1277

103 Mizoguchi, A. et al. (2002) Nectin: an adhesion molecule involved in formation of synapses. J Cell Biol 156, 555-565

104 Losh, M. et al. (2008) Defining key features of the broad autism phenotype: a comparison across parents of multiple- and single-incidence autism families. Am J Med Genet B Neuropsychiatr Genet 147B, 424-433

105 Virkud, Y.V. et al. (2009) Familial aggregation of quantitative autistic traits in multiplex versus simplex autism. Am J Med Genet B Neuropsychiatr Genet 150B, 328-334

106 Arking, D.E. et al. (2008) A common genetic variant in the neurexin superfamily member CNTNAP2 increases familial risk of autism. Am J Hum Genet 82, 160-164

107 Bonora, E. et al. (2005) Mutation screening and association analysis of six candidate genes for autism on chromosome 7q. Eur J Hum Genet 13, 198-207 
108 Sakurai, T. et al. (2006) Association analysis of the NrCAM gene in autism and in subsets of families with severe obsessive-compulsive or self-stimulatory behaviors. Psychiatr Genet 16, 251257

109 Marui, T. et al. (2009) Association of the neuronal cell adhesion molecule (NRCAM) gene variants with autism. Int J Neuropsychopharmacol 12, 1-10

110 Fatemi, S.H. (2005) Reelin glycoprotein: structure, biology and roles in health and disease. Mol Psychiatry 10, 251-257

111 Wang, K. et al. Common genetic variants on $5 \mathrm{p} 14.1$ associate with autism spectrum disorders. Nature 459, 528-533

112 Fiala, J.C. et al. (2002) Dendritic spine pathology: cause or consequence of neurological disorders? Brain Res Brain Res Rev 39, 29-54

113 Ethell, I.M. and Pasquale, E.B. (2005) Molecular mechanisms of dendritic spine development and remodeling. Prog Neurobiol 75, 161-205

114 Zoghbi, H.Y. (2003) Postnatal neurodevelopmental disorders: meeting at the synapse? Science $302,826-830$

115 Rubenstein, J.L. and Merzenich, M.M. (2003) Model of autism: increased ratio of excitation/inhibition in key neural systems. Genes Brain Behav 2, 255-267

116 Huang, Z.J. and Scheiffele, P. (2008) GABA and neuroligin signaling: linking synaptic activity and adhesion in inhibitory synapse development. Curr Opin Neurobiol 18, 77-83

117 Minshew, N.J. and Williams, D.L. (2007) The new neurobiology of autism: cortex, connectivity, and neuronal organization. Arch Neurol 64, 945-950

118 Reddy, K.S. (2005) Cytogenetic abnormalities and fragile-X syndrome in autism spectrum disorder. BMC Med Genet 6, 3

119 Clifford, S. et al. (2007) Autism spectrum phenotype in males and females with fragile $X$ full mutation and premutation. J Autism Dev Disord 37, 738-747

120 Dictenberg, J.B. et al. (2008) A direct role for FMRP in activity-dependent dendritic mRNA transport links filopodial-spine morphogenesis to fragile X syndrome. Dev Cell 14, 926-939

121 Bear, M.F. et al. (2004) The mGluR theory of fragile X mental retardation. Trends Neurosci 27, 370-377

122 Yan, Q.J. et al. (2005) Suppression of two major Fragile X Syndrome mouse model phenotypes by the mGluR5 antagonist MPEP. Neuropharmacology 49, 1053-1066

123 Dolen, G. et al. (2007) Correction of fragile X syndrome in mice. Neuron 56, 955-962

124 Nakamoto, M. et al. (2007) Fragile $X$ mental retardation protein deficiency leads to excessive mGluR5-dependent internalization of AMPA receptors. Proc Natl Acad Sci U S A 104, 1553715542

125 de Vrij, F.M. et al. (2008) Rescue of behavioral phenotype and neuronal protrusion morphology in Fmr1 KO mice. Neurobiol Dis 31, 127-132

126 Guy, J. et al. (2007) Reversal of neurological defects in a mouse model of Rett syndrome. Science 315, 1143-1147

127 Giacometti, E. et al. (2007) Partial rescue of MeCP2 deficiency by postnatal activation of MeCP2. Proc Natl Acad Sci U S A 104, 1931-1936

128 Tropea, D. et al. (2009) Partial reversal of Rett Syndrome-like symptoms in MeCP2 mutant mice. Proc Natl Acad Sci U S A 106, 2029-2034

129 Ehninger, D. et al. (2008) Reversal of learning deficits in a Tsc2+/- mouse model of tuberous sclerosis. Nat Med 14, 843-848

130 Zhou, J. et al. (2009) Pharmacological inhibition of mTORC1 suppresses anatomical, cellular, and behavioral abnormalities in neural-specific Pten knock-out mice. J Neurosci 29, 1773-1783

131 Zeng, L.H. et al. (2008) Rapamycin prevents epilepsy in a mouse model of tuberous sclerosis complex. Ann Neurol 63, 444-453 
132 Chow, D.K. et al. (2009) Laminar and compartmental regulation of dendritic growth in mature cortex. Nat Neurosci 12, 116-118

133 Schmidt, H. et al. (2009) Intranasal insulin to improve developmental delay in children with 22q13 deletion syndrome: an exploratory clinical trial. J Med Genet 46, 217-222

134 Reich, D.E. and Lander, E.S. (2001) On the allelic spectrum of human disease. Trends Genet 17, 502-510

135 Pritchard, J.K. (2001) Are rare variants responsible for susceptibility to complex diseases? Am J Hum Genet 69, 124-137

136 Pritchard, J.K. and Cox, N.J. (2002) The allelic architecture of human disease genes: common disease-common variant... or not? Hum Mol Genet 11, 2417-2423

137 Bodmer, W. and Bonilla, C. (2008) Common and rare variants in multifactorial susceptibility to common diseases. Nat Genet 40, 695-701

138 Friedman, J.I. et al. (2008) CNTNAP2 gene dosage variation is associated with schizophrenia and epilepsy. Mol Psychiatry 13, 261-266

139 Lachman, H.M. et al. (2008) Analysis of protocadherin alpha gene deletion variant in bipolar disorder and schizophrenia. Psychiatr Genet 18, 110-115

140 Brennaman, L.H. and Maness, P.F. (2008) NCAM in neuropsychiatric and neurodegenerative disorders. Neurochem Res Mar 132008 [Epub ahead of print]

141 O'Donovan, M.C. et al. (2008) Phenotypic variations on the theme of CNVs. Nat Genet 40, 13921393 
Betancur et al.

Table 1. Genes involved in synaptic cell-adhesion pathways implicated in ASDs and ID

\begin{tabular}{|c|c|c|c|c|c|c|}
\hline Gene & Name & $\begin{array}{l}\text { Chromosomal } \\
\text { locus }\end{array}$ & Evidence & Phenotype & Function & Reference \\
\hline \multicolumn{7}{|c|}{ Cell adhesion molecules } \\
\hline $\mathrm{CDH} / \mathrm{CDH} 10$ & Cadherin 9/Cadherin 10 & $5 p 14.1$ & common SNPs & ASD & CAM & [111] \\
\hline $\mathrm{CDH} 15$ & Cadherin 15 & $16 q 24.3$ & $\begin{array}{l}\text { chromosomal abnormality, } \\
\text { rare mutations }\end{array}$ & ID & CAM & [82] \\
\hline $\mathrm{CDH} 18$ & Cadherin 18 & $5 p 14.3$ & chromosomal abnormality & ASD & CAM & [4] \\
\hline CNTN3 & Contactin 3 & $3 p 12.3$ & homozygous deletion & ASD & CAM & [80] \\
\hline CNTN4 & Contactin 4 & $3 p 26.3$ & $\begin{array}{l}\text { deletion, chromosomal } \\
\text { abnormalities, CNVs }\end{array}$ & $3 p$ deletion syndrome, $A S D$, ID & CAM & {$[44,90,91]$} \\
\hline CNTNAP2 & Contactin associated protein 2 & $7 q 36.1$ & $\begin{array}{l}\text { rare mutations, deletion, } \\
\text { chromosomal abnormality, } \\
\text { common SNPs }\end{array}$ & $\begin{array}{l}\text { ASD, language deficits, schizophrenia, cortical } \\
\text { dysplasia, epilepsy, ID, Tourette syndrome, OCD }\end{array}$ & CAM & {$[94,97-100,106]$} \\
\hline KIRREL3 & Kin of IRRE like 3 & $11 q 24.2$ & $\begin{array}{l}\text { chromosomal abnormality, } \\
\text { rare mutations }\end{array}$ & ID & CAM & [82] \\
\hline L1CAM & L1 cell adhesion molecule & Xq28 & mutations, deletions & syndromic ID (hydrocephalus, spastic paraplegia) & CAM & [88] \\
\hline NLGN3 & Neuroligin 3 & Xq13.1 & rare mutations & ASD & CAM & [12] \\
\hline NLGN4X & Neuroligin 4 & $\begin{array}{l}\text { Xp22.32- } \\
\text { p22.31 }\end{array}$ & rare mutations, CNVs & $\begin{array}{l}\text { ASD, ID; in the case of deletions: Tourette } \\
\text { syndrome, ADHD, learning disorders, anxiety and } \\
\text { depression (including healthy carriers) }\end{array}$ & CAM & {$[4,12,17,25-28]$} \\
\hline NRCAM & $\begin{array}{l}\text { NgCAM related cell adhesion } \\
\text { molecule }\end{array}$ & $7 q 31.1$ & common SNPs & ASD & CAM & [107-109] \\
\hline$N R X N 1$ & Neurexin 1 & $2 p 16.3$ & $\begin{array}{l}\text { CNVs, chromosomal } \\
\text { abnormalities }\end{array}$ & ASD, schizophrenia, CNVs also in healthy carriers & CAM & {$[4,42-45,49]$} \\
\hline PCDH9 & Protocadherin 9 & $13 q 21.32$ & CNVs & ASD, CNVs also in healthy carriers & CAM & [4] \\
\hline PCDH10 & Protocadherin 10 & $4 q 28.3$ & homozygous deletion & ASD & CAM & [80] \\
\hline PCDH19 & Protocadherin 19 & $X q 22.1$ & rare mutations & $\begin{array}{l}\text { epilepsy, female-restricted, with mental retardation } \\
\text { (EFMR) }\end{array}$ & CAM & [81] \\
\hline \multicolumn{7}{|c|}{ Additional molecules involved in synaptic cell adhesion pathways } \\
\hline CASK & $\begin{array}{l}\text { Calcium/calmodulin-dependent } \\
\text { serine protein kinase }\end{array}$ & Xp11.4 & rare mutations, deletion & ID, brain malformation & $\begin{array}{l}\text { Scaffolding } \\
\text { protein/kinase }\end{array}$ & {$[55,56]$} \\
\hline CDK5R1 & $\begin{array}{l}\text { Cyclin-dependent kinase } 5 \text {, } \\
\text { regulatory subunit } 1\end{array}$ & $77 q 11.2$ & rare mutations & non syndromic ID & Kinase & [54] \\
\hline DLG1/PAK2 & $\begin{array}{l}\text { Discs large (Drosophila) homolog } \\
\text { 1/p21-activated kinase } 2\end{array}$ & $3 q 29$ & deletion & 3q29 microdeletion syndrome, ID, ASD & $\begin{array}{l}\text { Scaffolding } \\
\text { protein/kinase }\end{array}$ & [101] \\
\hline SHANK3 & $\begin{array}{l}\text { SH3 and multiple ankyrin repeat } \\
\text { domains } 3\end{array}$ & $22 q 13.3$ & $\begin{array}{l}\text { deletions, chromosomal } \\
\text { abnormalities, rare } \\
\text { mutations }\end{array}$ & $22 q 13$ deletion syndrome, ASD, ID & $\begin{array}{l}\text { Scaffolding } \\
\text { protein }\end{array}$ & {$[66-68,72,73]$} \\
\hline
\end{tabular}

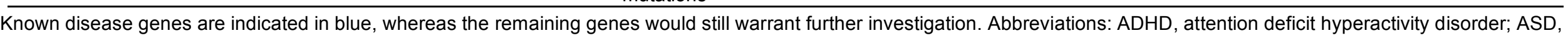
autism spectrum disorders; CAM, cell adhesion molecule; CNVs, copy number variations; ID, intellectual disability; OCD, obsessive compulsive disorder; SNPs, single nucleotide polymorphisms. 


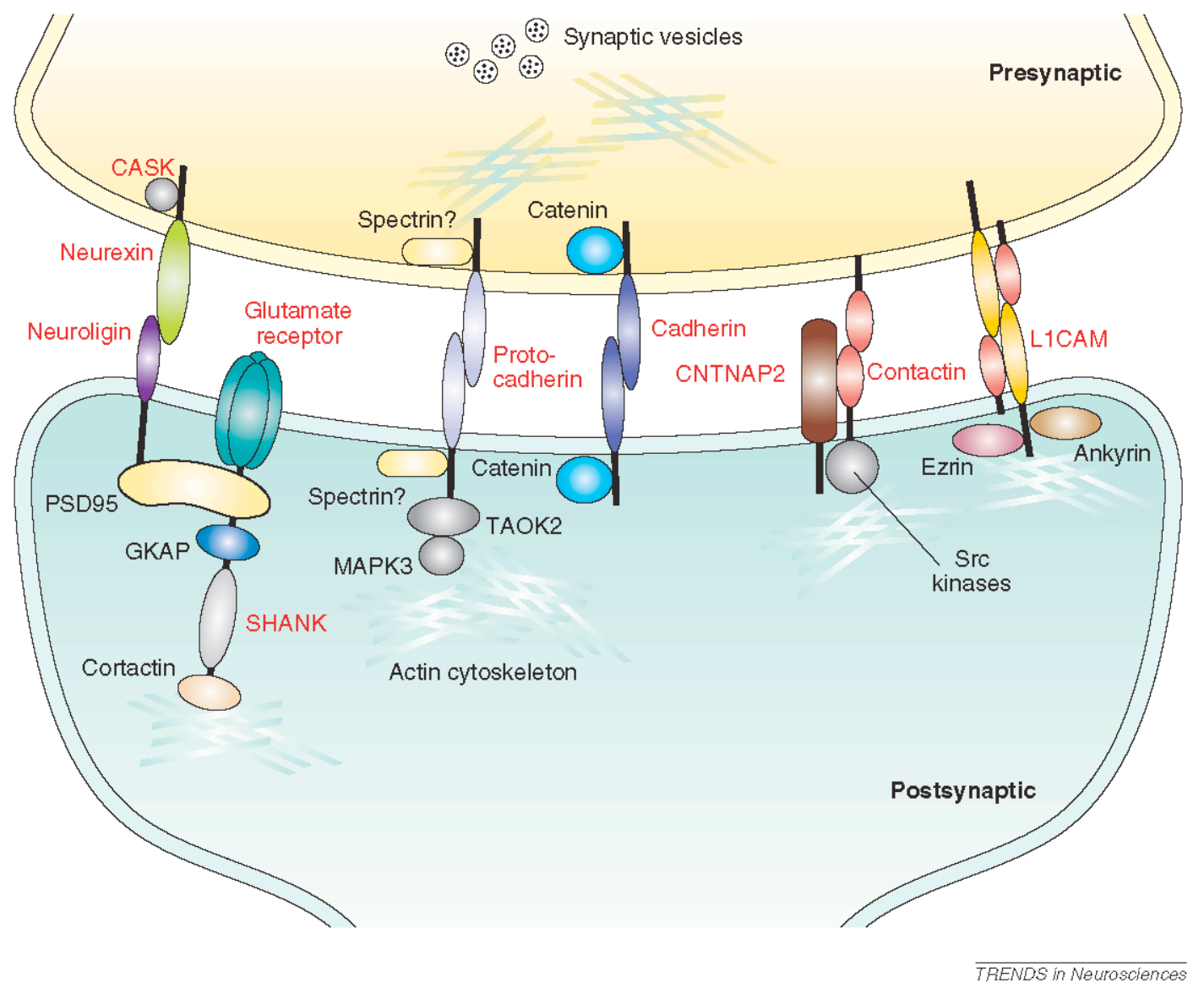

Figure 1. At the synapses, cell adhesion molecules connect presynaptic and postsynaptic sites through homophilic (e.g., caherins, protocadherins and L1CAM) and heterophilic (e.g., neuroliginneurexin, CNTNAP2 and contactins) interactions, and play roles in synapse development, function and plasticity. These cell adhesion molecules also interact with several cytoplasmic proteins, namely synaptic scaffolding proteins (e.g., postsynaptic density protein 95 [PSD95] and SHANK, which in turn serve as platforms for signaling molecules), cytoskeletal proteins (e.g., ankryin, ezrin, catenin, actin and spectrin) and signaling molecules (e.g., calmodulin-dependent serine kinase [CASK], src family kinases, and mitogen-activated protein kinase [MAPK] cascade proteins including TAO kinase 2 [TAOK2] and MAPK3), all of which are involved in synapse assembly and shape, function, and plasticity. Expression of cell adhesion molecules can determine the chemical nature of synapses and the downstream signaling. The expression and localization of cell adhesion molecules are modulated by neuronal activity, leading to regulation of synaptic strength and sculpting of the molecular components of the synapse. The proteins whose genes have been shown to be involved in ASD and/or ID are indicated in red. The question marks shown after spectrin highlight the fact that the functional meaning of the interaction between this protein and protocadherins is unclear. 
Box 1. The 'common disease-common variant' versus the 'multiple rare variants' perspectives

In many genetic diseases, including ASDs, there have been two models for explaining genetic etiology, which are not mutually exclusive. These are the 'common disease/common variant' and the 'common disease/rare variant' models, the latter also called the 'multiple rare variant' or 'rare alleles of major effect' model ${ }^{[134-137]}$. Common variants are genetic polymorphisms (susceptibility alleles) that are found widely distributed in the population and are associated with a modest increase in disease risk, typically associated with odds ratios (ORs) less than 2 (often in the $\sim 1-1.3$ range). In this model, a given disorder stems from the interaction of multiple common genetic variants and possibly other nongenetic factors. The common disease/common variant hypothesis has been challenged from a population genetic perspective because if one assumes any sort of selection against even moderately deleterious variants, the rates of the variants would be very low ${ }^{[134-136]}$. In contrast, in the multiple rare variant model, a large number of rare, and even very rare, variants underlie the disorder. These rare variants are typically highly penetrant, associated with ORs that can be substantial and may contribute the major part of the susceptibility for a given individual. At the extreme, rare variants have high ORs and are equivalent to rare deleterious mutations. These two models have very different implications regarding gene discovery strategies. While association studies are suitable to identify common susceptibility alleles present in large numbers of patients compared to controls, they fail to identify rare, causal mutations. Similarly, linkage studies are unable to identify mutations in critical genes in highly heterogeneous disorders involving many different genes and chromosomal loci such as ASDs.

\section{Box 2. ASD and schizophrenia genetics}

While ASDs are clearly distinct from schizophrenia, CNVs in identical genes have been identified in both disorders during the last years. For example, some of the synaptic CAMs noted in the current review have also been implicated in schizophrenia through CNVs and chromosomal abnormalities, including $N R X N 1^{[49]}$ and CNTNAP2 ${ }^{[138]}$, as well as other CAMs, including protocadherin $\alpha^{[139]}$ and NCAM ${ }^{[140]}$. Similarly, recent large-scale genome-wide studies identified CNVs at 1q21.1, 15q11.2, 15q13.3, and 16p11.2 in schizophrenia, ASD and ID. While at first surprising, these findings are not inconsistent with the well-known observation that the 22q11.2 deletion syndrome (velo-cardio-facial syndrome) increases risk for schizophrenia and for autism and suggest that neurodevelopmental abnormalities can lead to profoundly different manifestations depending on other factors that are as yet unknown. One can envision two scenarios ${ }^{[141]}$. In one case the CNV induces a mediating phenotype, such as cognitive impairment, which in turn increases risk for both ASD and schizophrenia. Alternatively, there are pleiotropic effects of the CNV without a mediating intermediate phenotype. Understanding the influence of CNVs and specific susceptibility genes in various clinical disorders will benefit from an explicit study of the relationship of the genetic variation to potential intermediate phenotypes, including detailed phenotypic assessment of apparently "unaffected" carriers. 